\title{
Incorporating Price-Responsive Demand in Energy Scheduling Based on Consumer Payment Minimization
}

\author{
Ricardo Fernández-Blanco, Member, IEEE, José M. Arroyo, Senior Member, IEEE, \\ Natalia Alguacil, Senior Member, IEEE, and Xiaohong Guan, Fellow, IEEE
}

\begin{abstract}
In the restructured power industry, demand responsiveness is a key factor whose importance will be boosted due to the impetus provided by the development of smart grids. Within the context of pool-based electricity markets, this paper addresses the incorporation of price-responsive demand in multiperiod energy scheduling driven by consumer payment minimization. Although consumer payment minimization has drawn considerable attention mainly in an operational setting and also recently under a planning framework, available models and solution approaches typically neglect demand-side participation. The proposed scheduling model considers a marginal pricing scheme as well as the effects of both network constraints and intertemporal constraints associated with generation operation. Modeling demand-side participation leads to bilinear payment terms that significantly increase the mathematical complexity of the optimization process. The resulting problem is formulated as a mixed-integer nonlinear bilevel program for which no exact solution technique is currently available. This paper presents a novel methodology by which the original bilevel and bilinear problem is converted into an equivalent single-level mixed-integer linear program suitable for efficient off-the-shelf software. This transformation is based on the application of duality theory of linear programming, integer algebra, and Karush-Kuhn-Tucker optimality conditions. The proposed approach has been successfully applied to the IEEE 118-bus system.
\end{abstract}

Index Terms-Bilevel programming, bilinear terms, consumer payment minimization, demand response, energy scheduling, marginal pricing, mixed-integer linear equivalent.

Manuscript received September 23, 2014; revised January 23, 2015 and April 13, 2015; accepted April 26, 2015. Date of publication June 18, 2015; date of current version February 17, 2016. This work was supported in part by the Ministry of Science of Spain under CICYT Project ENE2012-30679, in part by the European Commission under Grant 309048 "SiNGULAR", in part by the Junta de Comunidades de Castilla-La Mancha under Project POII-2014-012-P, and in part by the Universidad de Castilla-La Mancha under Grant GI20152945. Paper no. TSG-00946-2014.

R. Fernández-Blanco is with the Department of Electrical Engineering, University of Washington, Seattle, WA 98195-2500 USA (e-mail: ricardo.fcarramolino@gmail.com).

J. M. Arroyo and N. Alguacil are with the Departamento de Ingeniería Eléctrica, Electrónica, Automática y Comunicaciones, E.T.S.I. Industriales, Universidad de Castilla-La Mancha, Ciudad Real E-13071, Spain (e-mail: josemanuel.arroyo@uclm.es; natalia.alguacil@uclm.es).

$\mathrm{X}$. Guan is with the Systems Engineering Institute, SKLMSE Laboratory, and MOE KLINNS Laboratory, Xi'an Jiaotong University, Xi'an 710049, China, and also with the Center for Intelligent and Networked Systems and TNLIST, Tsinghua University, Beijing 100084, China (e-mail: xhguan@sei.xjtu.edu.cn).

Digital Object Identifier 10.1109/TSG.2015.2427584

\section{NOMENCLATURE}

\section{Indices}

$\begin{array}{ll}b & \text { Demand bid block. } \\ i & \text { Generating unit. } \\ j & \text { Consumer. } \\ l & \text { Transmission line. } \\ n & \text { Bus. } \\ n(i) & \text { Bus where generating unit } i \text { is located. } \\ n(j) & \text { Bus where consumer } j \text { is located. } \\ o & \text { Generation offer block. } \\ t & \text { Time period. }\end{array}$

Sets

$\mathcal{B}_{j} \quad$ Indices of the demand bid blocks of consumer $j$.

$\mathcal{F}_{i t} \quad$ Feasibility set for the scheduling variables associated with unit $i$ in period $t$.

$\mathcal{I} \quad$ Indices of generating units.

$\mathcal{I}_{n} \quad$ Indices of the generating units located at bus $n$.

$\mathcal{J} \quad$ Indices of consumers.

$\mathcal{J}_{n} \quad$ Indices of the consumers located at bus $n$.

$\mathcal{L}_{t}^{+} \quad$ Indices of transmission lines for the constraints imposing the upper bounds on line power flows in period $t$.

$\mathcal{L}_{t}^{-} \quad$ Indices of transmission lines for the constraints imposing the lower bounds on line power flows in period $t$.

$\mathcal{N} \quad$ Indices of buses.

$\mathcal{O}_{i} \quad$ Indices of the generation offer blocks of unit $i$.

$\mathcal{T} \quad$ Indices of time periods.

\section{Constants}

$A_{l n} \quad$ Sensitivity factor for line $l$ and bus $n$.

$C_{b j t}^{d} \quad$ Price of the $b$ th demand block bid by consumer $j$ in period $t$.

$C_{\text {oit }}^{g} \quad$ Price of the $o$ th generation block offered by unit $i$ in period $t$.

$n_{B_{j}} \quad$ Cardinality of $\mathcal{B}_{j}$.

$n_{I} \quad$ Number of generating units.

$n_{J} \quad$ Number of consumers.

$n_{L_{t}^{+}} \quad$ Cardinality of $\mathcal{L}_{t}^{+}$.

$n_{L_{t}^{-}} \quad$ Cardinality of $\mathcal{L}_{t}^{-}$.

$n_{N} \quad$ Number of buses.

$n_{O_{i}} \quad$ Cardinality of $\mathcal{O}_{i}$.

$n_{T} \quad$ Number of time periods. 


\begin{tabular}{|c|c|c|}
\hline $\bar{P}_{b j t}^{d}$ & Upper bound for $p_{b j t}^{d}$. & $\lambda_{t}$ \\
\hline$P_{j t}^{d}$ & Lower bound for $p_{j t}^{d}$. & \\
\hline $\bar{P}_{j t}^{d}$ & Upper bound for $p_{j t}^{d}$. & \\
\hline $\bar{P}_{l}^{f}$ & Power flow capacity of line $l$. & $\mu_{b i t}^{\text {up }}$ \\
\hline$P_{i 0}^{g}$ & Initial power output of unit $i$. & \\
\hline$\underline{P}_{i t}^{g}$ & Lower bound for $p_{i t}^{g}$ & $\xi_{i t}$ \\
\hline $\bar{P}_{i t}^{g}$ & Upper bound for $p_{i t}^{g}$. & \\
\hline $\bar{P}_{\text {oit }}^{g}$ & Upper bound for $p_{\text {oit }}^{g}$. & \\
\hline$R_{i}^{\mathrm{dn}}$ & Ramp-down rate of unit $i$. & $\pi_{n t}$ \\
\hline$R_{i}^{\text {sd }}$ & Shut-down ramp rate of unit $i$. & \\
\hline$R_{i}^{\mathrm{su}}$ & Start-up ramp rate of unit $i$ & \\
\hline$R_{i}^{\mathrm{up}}$ & Ramp-up rate of unit $i$ & \\
\hline$V_{i 0}$ & Initial on/off status of unit $i$. & \\
\hline$\underline{\gamma}_{i t}^{1 \mathrm{lo}}$ & Lower bound for $\gamma_{i t}^{\mathrm{lo}}$ & $\sigma_{i}$ \\
\hline $\bar{\gamma}_{i t}^{\text {up }}$ & Upper bound for $\gamma_{i t}^{\text {up }}$. & \\
\hline $\bar{\epsilon}_{i t}$ & Upper bound for $\epsilon_{i t}$. & $\phi_{l t}^{100}$ \\
\hline $\bar{\xi}_{i t}$ & Upper bound for $\xi_{i t}$. & \\
\hline$\underline{\sigma}_{i t}$ & Lower bound for $\sigma_{i t}$. & \\
\hline
\end{tabular}

\section{Variables}

$a_{i t} \quad$ Auxiliary variable equal to the product $v_{i t} \gamma_{i t}^{\text {lo }}$.

$b_{i t} \quad$ Auxiliary variable equal to the product $v_{i t} \gamma_{i t}^{\text {up }}$.

$c_{i t} \quad$ Auxiliary variable equal to the product $v_{i t} \xi_{i t}$.

$d_{i t} \quad$ Auxiliary variable equal to the product $v_{i t-1} \xi_{i t}$.

$e_{i t} \quad$ Auxiliary variable equal to the product $v_{i t} \epsilon_{i t}$.

$k_{i t} \quad$ Auxiliary variable equal to the product $v_{i t+1} \epsilon_{i t}$.

$m_{i t} \quad$ Auxiliary variable equal to the product $v_{i t} \sigma_{i t}$.

$p_{b j t}^{d} \quad$ Consumption level awarded to block $b$ bid by consumer $j$ in period $t$.

$p_{j t}^{d} \quad$ Power consumption of consumer $j$ in period $t$.

$p_{i t}^{g} \quad$ Power output of unit $i$ in period $t$.

$p_{\text {oit }}^{g} \quad$ Generation level awarded to block $o$ offered by unit $i$ in period $t$.

$r_{i t}$

$s_{i t}$

$v_{i t}$

$\alpha_{i t}$

Auxiliary variable equal to the product $v_{i t-1} \sigma_{i t}$.

Payment for the start-up of unit $i$ in period $t$.

On/off status of unit $i$ in period $t$ that is equal

to 1 if unit $i$ is scheduled on in period $t$, being 0 otherwise.

Dual variable associated with the definition of $p_{i t}^{g}$ as the sum of the generation levels awarded to the offer blocks.

$\beta_{\text {oit }}^{\text {lo }} \quad$ Dual variable associated with the constraint imposing the lower bound for $p_{\text {oit }}^{g}$.

$\beta_{\text {oit }}^{\text {up }} \quad$ Dual variable associated with the constraint imposing the upper bound for $p_{\text {oit }}^{g}$.

$\gamma_{i t}^{\text {lo }} \quad$ Dual variable associated with the constraint imposing the lower bound for $p_{i t}^{g}$.

$\gamma_{i t}^{\text {up }} \quad$ Dual variable associated with the constraint imposing the upper bound for $p_{i t}^{g}$.

$\delta_{j t} \quad$ Dual variable associated with the definition of $p_{j t}^{d}$ as the sum of the consumption levels awarded to the demand bid blocks.

$\epsilon_{i t} \quad$ Dual variable associated with the shut-down ramp rate constraint of unit $i$ in period $t$. $\lambda_{t} \quad$ Dual variable associated with the power balance equation in period $t$.

Dual variable associated with the constraint imposing the lower bound for $p_{b j t}^{d}$.

Dual variable associated with the constraint imposing the upper bound for $p_{b j t}^{d}$.

Dual variable associated with the constraint modeling the ramp-up and start-up ramp rates of unit $i$ in period $t$.

Locational marginal price (LMP) at bus $n$ in period $t$.

Dual variable associated with the constraint imposing the lower bound for $p_{j t}^{d}$.

Dual variable associated with the constraint imposing the upper bound for $p_{j t}^{d}$.

Dual variable associated with the ramp-down rate constraint of unit $i$ in period $t$.

Dual variable associated with the constraint imposing the lower bound for the power flow of line $l$ in period $t$.

Dual variable associated with the constraint imposing the upper bound for the power flow of line $l$ in period $t$.

\section{INTRODUCTION}

Q INCE THE pioneering work of Jacobs [1], consumer $\checkmark$ payment minimization has triggered extensive research in the fields of energy scheduling [2]-[15] and investment planning [16]. However, most works have neglected the effect of demand response, which is crucial for guaranteeing the efficiency and competitiveness of the restructured power industry [17]-[24]. The advent of smart grids provides a suitable framework to boost the long-desired participation of demand at both wholesale market level [17]-[19], [21] and distribution level [20], [22]-[24]. Therefore, the new context where smart grids come into play calls for changes in the operational and planning models that have typically disregarded demand-side participation such as those wherein the consumer payment is minimized [2]-[4], [7]-[9], [11]-[16].

One of those problems is wholesale energy scheduling based on consumer payment minimization in pool-based electricity markets [2]-[4], [7]-[9], [11]-[15]. In the presence of price-responsive demand, the solution to this problem provides the system operator with the set of awarded generation offers and demand bids that yields the minimum payment to consumers. Such market-clearing procedure is implemented while meeting the operational constraints associated with electricity generation and consumption and keeping the privacy of the information submitted by market participants. Energy scheduling driven by consumer payment minimization has been proposed as a remedy to a practical issue in pool-based electricity markets, namely the lack of incentives for suppliers to offer their actual production costs, which are private corporate information. Thus, consumer payment minimization can be viewed as an instrument to protect consumers against dishonest behavior and market power abuse by generation companies. 
Rather than discussing the suitability of consumer payment minimization as an adequate optimization goal for wholesale energy scheduling, this paper addresses the challenges and issues associated with the consideration of price-responsive demand, i.e., demand-side bidding, in such operational problem. From a mathematical viewpoint, this practical aspect gives rise to a major complicating feature. Since the consumer payment is defined in terms of products of energy prices and consumption levels, energy prices become decision variables of the optimization process, thereby requiring an explicit mathematical characterization in the problem formulation. Moreover, incorporating demand responsiveness implies extending the set of decision variables to include consumption levels. Thus, the aforementioned payment terms involve products of two continuous variables. It is worth emphasizing that no exact general methodology is currently available to deal with the resulting optimization problem involving such bilinear and highly nonconvex terms.

To the best of the authors' knowledge, demand-side participation has only been accounted for in the framework of consumer payment minimization in [5], [6], and [10]. Demand bids were theoretically considered in the two-layer structure presented in [5] to characterize LMPs but no solution methodology was provided. In [6] and [10], demand-side bidding was also taken into account in the consumer payment minimization problem. However, rather than standard marginal pricing [25], [26], both models adopted alternative pricing schemes which may be of reduced practical applicability. Hence, new models and methodologies are yet to be explored.

The thrust of this paper is the proposal of a novel approach for multiperiod energy scheduling based on consumer payment minimization wherein demand-side bidding is explicitly considered under the widely used marginal pricing scheme. As done in [8] and [15] for the case with inelastic demand, a bilevel programming framework [27] is used to properly account for marginal pricing. The schedule of generating units is determined in the upper-level problem, whereas generation and consumption levels result from the lower-level problem. In addition, dual information from the lower level is used to compute LMPs in the upper level. The resulting problem is formulated as a mixed-integer nonlinear bilevel program with bilinear terms. The presence of bilinear payment terms constitutes the main distinctive modeling aspect with respect to the models described in [8] and [15]. As above mentioned, such salient feature renders the problem unsuitable for existing solution techniques.

The key idea of the proposed solution approach is to convert the original mixed-integer nonlinear bilevel problem into an equivalent single-level mixed-integer linear program. To that end, a two-step procedure is implemented. First, similar to [15], practical modeling aspects allow applying an effective primal-dual transformation [28] and integer algebra results [29] to recast the bilevel scheduling model as a single-level equivalent. It is worth mentioning that, unlike in [15], the resulting single-level optimization is a mixedinteger program with bilinear payment terms for which a global solution method is also unavailable. As a distinctive methodological feature of the proposed approach, a novel, exact, and computationally inexpensive linearization scheme based on duality theory of linear programming and KarushKuhn-Tucker (KKT) optimality conditions is subsequently applied to yield an equivalent single-level mixed-integer linear program. Mixed-integer linear programming guarantees finite convergence to optimality [30]. Moreover, efficient off-theshelf branch-and-cut software is available [31].

This paper thus features three major novelties with respect to previous works on wholesale energy scheduling driven by consumer payment minimization [2]-[15].

1) Unlike [2]-[4], [7]-[9], and [11]-[15], demand-side bidding is explicitly accounted for.

2) In contrast to [6] and [10], the model includes locational marginal pricing and intertemporal operational constraints.

3) A sound and effective solution approach is proposed.

More specifically, the main contributions of this paper are threefold.

1) From a modeling perspective, a novel formulation for energy scheduling driven by consumer payment minimization under marginal pricing is presented wherein price-responsive demand is explicitly incorporated. The resulting model, which includes transmission network constraints, intertemporal operational constraints, and marginal pricing, is formulated as a mixed-integer nonlinear bilevel program with bilinear terms.

2) From a methodological perspective, this paper provides a novel and exact linearization of the bilinear terms associated with the consumer payment for energy. Such linearization, in conjunction with a previously reported primal-dual transformation and well-known integer algebra results, allows formulating the original bilevel problem with bilinear terms as a single-level mixed-integer linear equivalent, with no additional binary variables, for which efficient solvers exist.

3) Numerical experience is reported from solving a case study significantly larger than those analyzed in previous works addressing demand-side participation in the consumer payment minimization problem.

The ultimate purpose of this paper is to provide the system operator and market agents with a tool that allows a comprehensive assessment of energy scheduling driven by consumer payment minimization so that informed decisions can be made on its eventual implementation in real-world poolbased electricity markets. Moreover, the proposed approach may be useful for the system operator and the regulator in two respects, namely: 1) to monitor the behavior of market agents when the consumer payment is minimized under marginal pricing; and 2) to examine the short- and long-term implications of this trading scheme. Such analyses, which are beyond the scope of this paper, may require dynamic game theoretical models or equilibrium-based models wherein the proposed tool would play a key role. Relevant related works are those by Vázquez et al. [4] and Zhao et al. [9], where simplified models and approaches disregarding demand-side participation were used.

The remainder of this paper is outlined as follows. Section II presents the formulation for the scheduling model with 
price-responsive demand. In Section III, the proposed solution approach is described. Numerical results are discussed in Section IV. Finally, the conclusion is drawn in Section V.

\section{PROBlem Formulation}

This section presents the mathematical formulation for the network-constrained multiperiod energy scheduling problem driven by consumer payment minimization with priceresponsive demand under marginal pricing. Within the context of current industry practice regarding demand-side bidding in pool-based electricity markets [32]-[34], the focus is placed on demand price responsiveness in the form of bids including pairs quantity-price and bounds on consumption levels. This model is simpler to describe and analyze, yet bringing out the issue of demand-side participation in the consumer payment minimization problem. Based on [8] and [15], the proposed auction model can be formulated as the following bilevel program:

$$
\underset{s_{i t}, v_{i t}, \pi_{n t}}{\operatorname{Minimize}} \sum_{j \in \mathcal{J}} \sum_{t \in \mathcal{T}} \pi_{n(j) t} p_{j t}^{d}+\sum_{i \in \mathcal{I}} \sum_{t \in \mathcal{T}} s_{i t}
$$

subject to

$$
\begin{array}{ll}
\pi_{n t}=\lambda_{t}-\sum_{l \in \mathcal{L}_{t}^{+}} A_{l n} \phi_{l t}^{\text {up }} & \\
-\sum_{l \in \mathcal{L}_{t}^{-}} A_{l n} \phi_{l t}^{\text {lo }} ; & \forall n \in \mathcal{N}, \forall t \in \mathcal{T} \\
s_{i t}, v_{i t} \in \mathcal{F}_{i t} ; & \forall i \in \mathcal{I}, \quad \forall t \in \mathcal{T} \\
v_{i t} \in\{0,1\} ; & \forall i \in \mathcal{I}, \quad \forall t \in \mathcal{T}
\end{array}
$$

where $p_{j t}^{d}, \lambda_{t}, \phi_{l t}^{\text {up }}$, and $\phi_{l t}^{\mathrm{lo}}$ are obtained from the following multiperiod de optimal power flow:

$$
\begin{aligned}
& \underset{\substack{p_{b j t}^{d}, p_{j t}^{d}, \operatorname{Maximize}}}{ } \sum_{j \in \mathcal{J}} \sum_{b \in \mathcal{B}_{j}} \sum_{t \in \mathcal{T}} C_{b j t}^{d} p_{b j t}^{d}-\sum_{i \in \mathcal{I}} \sum_{o \in \mathcal{O}_{i}} \sum_{t \in \mathcal{T}} C_{o i t}^{g} p_{o i t}^{g} \\
& p_{i t}^{g}, p_{\text {oit }}^{g} \\
& \text { subject to } \\
& \sum_{j \in \mathcal{J}} p_{j t}^{d}=\sum_{i \in \mathcal{I}} p_{i t}^{g}:\left(\lambda_{t}\right) ; \quad \forall t \in \mathcal{T} \\
& \sum_{n \in \mathcal{N}} A_{l n}\left(\sum_{i \in \mathcal{I}_{n}} p_{i t}^{g}-\sum_{j \in \mathcal{J}_{n}} p_{j t}^{d}\right) \leq \bar{P}_{l}^{f}:\left(\phi_{l t}^{\mathrm{up}}\right) \\
& \forall t \in \mathcal{T}, \forall l \in \mathcal{L}_{t}^{+} \\
& \sum_{n \in \mathcal{N}} A_{l n}\left(\sum_{i \in \mathcal{I}_{n}} p_{i t}^{g}-\sum_{j \in \mathcal{J}_{n}} p_{j t}^{d}\right) \geq-\bar{P}_{l}^{f}:\left(\phi_{l t}^{\mathrm{lo}}\right) \\
& \forall t \in \mathcal{T}, \forall l \in \mathcal{L}_{t}^{-} \\
& \underline{P}_{i t}^{g} v_{i t} \leq p_{i t}^{g} \leq \bar{P}_{i t}^{g} v_{i t}:\left(\gamma_{i t}^{\text {lo }}, \gamma_{i t}^{\text {up }}\right) ; \quad \forall i \in \mathcal{I}, \forall t \in \mathcal{T} \\
& \underline{P}_{j t}^{d} \leq p_{j t}^{d} \leq \bar{P}_{j t}^{d}:\left(\rho_{j t}^{\mathrm{lo}}, \rho_{j t}^{\mathrm{up}}\right) ; \quad \forall j \in \mathcal{J}, \forall t \in \mathcal{T} \\
& p_{i t}^{g}=\sum_{o \in \mathcal{O}_{i}} p_{o i t}^{g}:\left(\alpha_{i t}\right) ; \quad \forall i \in \mathcal{I}, \forall t \in \mathcal{T} \\
& 0 \leq p_{\text {oit }}^{g} \leq \bar{P}_{\text {oit }}^{g}:\left(\beta_{\text {oit }}^{\text {lo }}, \beta_{\text {oit }}^{\text {up }}\right) ; \quad \forall i \in \mathcal{I}, \forall o \in \mathcal{O}_{i}, \\
& p_{j t}^{d}=\sum_{b \in \mathcal{B}_{j}} p_{b j t}^{d}:\left(\delta_{j t}\right) \\
& \forall j \in \mathcal{J}, \forall t \in \mathcal{T} \quad \text { (13) }
\end{aligned}
$$$$
\forall t \in \mathcal{T}(12)
$$

$$
\begin{aligned}
& 0 \leq p_{b j t}^{d} \leq \bar{P}_{b j t}^{d}:\left(\mu_{b j t}^{\mathrm{lo}}, \mu_{b j t}^{\mathrm{up}}\right) ; \quad \forall j \in \mathcal{J}, \forall b \in \mathcal{B}_{j}, \\
& p_{i t}^{g} \leq p_{i t-1}^{g}+R_{i}^{\mathrm{up}} v_{i t-1}+R_{i}^{\mathrm{su}}\left(v_{i t}-v_{i t-1}\right) \\
& +\bar{P}_{i t}^{g}\left(1-v_{i t}\right):\left(\xi_{i t}\right) ; \quad \forall i \in \mathcal{I}, \forall t \in \mathcal{T} \\
& p_{i t}^{g} \geq p_{i t-1}^{g}-R_{i}^{\mathrm{dn}} v_{i t}-R_{i}^{\mathrm{sd}}\left(v_{i t-1}-v_{i t}\right) \\
& -\bar{P}_{i t}^{g}\left(1-v_{i t-1}\right):\left(\sigma_{i t}\right) ; \quad \forall i \in \mathcal{I}, \forall t \in \mathcal{T} \\
& p_{i t}^{g} \leq R_{i}^{\mathrm{sd}}\left(v_{i t}-v_{i t+1}\right)+\bar{P}_{i t}^{g} v_{i t+1}:\left(\epsilon_{i t}\right) \forall i \in \mathcal{I}, \\
& \forall t=1 \ldots n_{T}-1 .
\end{aligned}
$$$$
\forall t \in \mathcal{T}(14)
$$

For the sake of consistency, hourly time periods are considered; $\sum_{b \in \mathcal{B}_{j}} \bar{P}_{b j t}^{d}=\bar{P}_{j t}^{d}, \forall j \in \mathcal{J}, \forall t \in \mathcal{T}$; and $\sum_{o \in \mathcal{O}_{i}} \bar{P}_{\text {oit }}^{g}=$ $\bar{P}_{i t}^{g}, \forall i \in \mathcal{I}, \forall t \in \mathcal{T}$.

The adoption of a marginal pricing scheme motivates the bilevel programming structure of (1)-(17). Under marginal pricing [25], locational energy prices for a given feasible schedule, represented by variables $v_{i t}$, are characterized in terms of the Lagrange multipliers or dual variables associated with a multiperiod optimal power flow wherein the declared social welfare is maximized once scheduling variables $v_{i t}$ have been fixed [35].

Moreover, based on [5], [6], and [10] and practical auction designs [32]-[34], demand-side participation is limited to price-responsive bids with consumption bounds. Notwithstanding, the mathematical structure of the proposed market-clearing procedure would remain essentially unaltered should other forms of demand response be modeled including on/off consumption scheduling, limited energy consumption over the scheduling horizon, consumption ramping limits, load shifting, operational consumption cycles, time-coupled price elasticity, among others [18]-[24].

The proposed bilevel model (1)-(17) consists of two optimization levels, namely the upper level (1)-(4) and the lower level (5)-(17). Upper-level variables are $s_{i t}$ and $v_{i t}$, associated with start-up offers and on/off statuses of generating units, respectively, as well as LMPs $\pi_{n t}$. Lower-level variables are power outputs $p_{i t}^{g}$, power consumptions $p_{j t}^{d}$, and awarded levels of generation offer and demand bid blocks $p_{\text {oit }}^{g}$ and $p_{b j t}^{d}$, respectively. Note that Lagrange multipliers or dual variables of the lower level are shown in parentheses in (6)-(17).

The upper-level problem minimizes the consumer payment (1), which comprises two terms. The first term represents the energy payment, which, as a major complicating feature, includes bilinear terms involving the products of two continuous variables, namely LMPs and consumption levels. The second term is related to the payment for start-ups. As done in [5], [7]-[9], and [11]-[15], start-up offers are fully compensated in (1).

According to [26], LMPs are defined in (2), where the first term in the right-hand side represents the energy component and the other two terms are the congestion components. Energy and congestion components correspond to the Lagrange multipliers or dual variables associated with the power balance equations and line capacity constraints, respectively, in the lower-level problem. As described in [36], sets $\mathcal{L}_{t}^{+}$and $\mathcal{L}_{t}^{-}$only include the indices of those lines that are prone to experience congestion as determined by 
a computationally inexpensive screening performed prior to the optimization process. Expressions (3) characterize generation constraints exclusively involving scheduling variables, i.e., minimum up and down times as well as start-ups. A detailed description of these linear constraints can be found in [37]. Expressions (4) set the integrality of the scheduling variables $v_{i t}$.

In contrast, the lower-level problem maximizes the declared social welfare (5) through a multiperiod optimal power flow particularized in terms of the scheduling variables $v_{i t}$. As is customary in day-ahead energy scheduling [8], [35], [36], [38]-[41], a dc load flow model is used to characterize the behavior of the transmission network, recognizing that the use of such a simplified model leads to results that may be optimistic and that a complete study of the scheduling problem should also consider the effect of reactive power. This generalization would, however, render the problem essentially intractable. This modeling limitation notwithstanding, the solution of the energy scheduling problem based on the dc load flow is acceptable for the purposes of the day-ahead operation of power systems [8], [35], [36], [38]-[41].

Using a model relying on linear sensitivity factors [39], the effect of the transmission network is considered in (6)-(8). Constraints (6) represent the power balance equations. As shown in [36], expressions (7) and (8) guarantee the fulfillment of line capacity limits across the transmission network. Note that the cardinalities of $\mathcal{L}_{t}^{+}$and $\mathcal{L}_{t}^{-}$are less than the number of transmission lines. Hence, the dimension of the model is reduced, thereby leading to computational savings. The interested reader is referred to [36] for the proof of the equivalence between this model and that considering power flow limits for the whole set of transmission lines. Production and consumption limits are set in (9) and (10), respectively. The block structures of generation offers and demand bids are characterized through constraints (11)-(12) and (13)-(14), respectively. Finally, ramping rates are modeled by (15)-(17) as described in [37].

\section{SOlution APPROACH}

The proposed solution approach consists in reformulating the original nonlinear bilevel program (1)-(17) as an equivalent single-level mixed-integer linear programming problem. To that end, duality theory of linear programming, integer algebra, and KKT optimality conditions are used as described next.

\section{A. Mixed-Integer Linear Equivalent for the Lower-Level Problem}

Based on the findings of [28], the lower-level problem can be equivalently replaced by its primal feasibility constraints (6)-(17), its dual feasibility constraints, and the equality corresponding to the strong duality theorem wherein the primal and dual objective functions of the lower-level problem are equated. It should be noted that duality theory of linear programming applies here since the lower-level optimization problem (5)-(17) is linear in the continuous lower-level primal variables $p_{b j t}^{d}, p_{j t}^{d}, p_{i t}^{g}, p_{\text {oit }}^{g}$, and since binary variables $v_{i t}$, determined by the upper-level problem, can be considered as parameters in the lower-level problem.
Lower-level dual feasibility constraints are given by

$$
\begin{aligned}
& -\lambda_{t}+\sum_{l \in \mathcal{L}_{t}^{+}} A_{\ln (i)} \phi_{l t}^{\mathrm{up}}+\sum_{l \in \mathcal{L}_{t}^{-}} A_{\ln (i)} \phi_{l t}^{\mathrm{lo}}+\gamma_{i t}^{\mathrm{lo}}+\gamma_{i t}^{\mathrm{up}} \\
& +\xi_{i t}-\xi_{i t+1}+\sigma_{i t}-\sigma_{i t+1}+\epsilon_{i t}+\alpha_{i t}=0 ; \\
& \forall i \in \mathcal{I}, \forall t=1 \ldots n_{T}-1 \\
& -\lambda_{n_{T}}+\sum_{l \in \mathcal{L}_{t}^{+}} A_{\ln (i)} \phi_{\ln T}^{\mathrm{up}}+\sum_{l \in \mathcal{L}_{t}^{-}} A_{\ln (i)} \phi_{\ln _{T}}^{\mathrm{lo}}+\gamma_{i n_{T}}^{\mathrm{lo}} \\
& +\gamma_{i n_{T}}^{\mathrm{up}}+\xi_{i n_{T}}+\sigma_{i n_{T}}+\alpha_{i n_{T}}=0 ; \quad \forall i \in \mathcal{I} \\
& -\alpha_{i t}+\beta_{\text {oit }}^{\text {lo }}+\beta_{\text {oit }}^{\text {up }}=-C_{\text {oit }}^{g} ; \quad \forall i \in \mathcal{I}, \forall o \in \mathcal{O}_{i}, \forall t \in \mathcal{T} \\
& \lambda_{t}+\delta_{j t}-\sum_{l \in \mathcal{L}_{t}^{+}} A_{l n(j)} \phi_{l t}^{\mathrm{up}}-\sum_{l \in \mathcal{L}_{t}^{-}} A_{\ln (j)} \phi_{l t}^{\mathrm{lo}} \\
& +\rho_{j t}^{\mathrm{lo}}+\rho_{j t}^{\mathrm{up}}=0 ; \quad \forall j \in \mathcal{J}, \forall t \in \mathcal{T} \\
& -\delta_{j t}+\mu_{b j t}^{\mathrm{lo}}+\mu_{b j t}^{\mathrm{up}}=C_{b j t}^{d} ; \quad \forall j \in \mathcal{J}, \forall b \in \mathcal{B}_{j}, \forall t \in \mathcal{T} \\
& \gamma_{i t}^{\text {lo }}, \sigma_{i t} \leq 0, \gamma_{i t}^{\text {up }}, \xi_{i t} \geq 0 ; \quad \forall i \in \mathcal{I}, \forall t \in \mathcal{T} \\
& \epsilon_{i t} \geq 0 \text {; } \\
& \rho_{j t}^{\text {lo }} \leq 0, \rho_{j t}^{\text {up }} \geq 0 \\
& \forall i \in \mathcal{I}, \forall t=1 \ldots n_{T}-1 \\
& \forall j \in \mathcal{J}, \forall t \in \mathcal{T} \\
& \phi_{l t}^{\mathrm{up}} \geq 0 \text {; } \\
& \forall t \in \mathcal{T}, \forall l \in \mathcal{L}_{t}^{+} \\
& \phi_{l t}^{\text {lo }} \leq 0 \text {; } \\
& \forall t \in \mathcal{T}, \forall l \in \mathcal{L}_{t}^{-} \\
& \beta_{\text {oit }}^{\text {lo }} \leq 0, \beta_{\text {oit }}^{\text {up }} \geq 0 \text {; } \\
& \forall i \in \mathcal{I}, \forall o \in \mathcal{O}_{i}, \forall t \in \mathcal{T} \\
& \mu_{b j t}^{\text {lo }} \leq 0, \mu_{b j t}^{\text {up }} \geq 0 \text {; } \\
& \forall j \in \mathcal{J}, \forall b \in \mathcal{B}_{j}, \forall t \in \mathcal{T}
\end{aligned}
$$

while the equality associated with the strong duality theorem is formulated as

$$
\begin{aligned}
& \sum_{j \in \mathcal{J}} \sum_{b \in \mathcal{B}_{j}} \sum_{t \in \mathcal{T}} C_{b j t}^{d} p_{b j t}^{d}-\sum_{i \in \mathcal{I}} \sum_{o \in \mathcal{O}_{i}} \sum_{t \in \mathcal{T}} C_{o i t}^{g} p_{o i t}^{g} \\
& =\sum_{t \in \mathcal{T}} \sum_{l \in \mathcal{L}_{t}^{+}} \bar{P}_{l}^{f} \phi_{l t}^{\text {up }}-\sum_{t \in \mathcal{T}} \sum_{l \in \mathcal{L}_{t}^{-}} \bar{P}_{l}^{f} \phi_{l t}^{\text {lo }} \\
& +\sum_{i \in \mathcal{I}} \sum_{t \in \mathcal{T}}\left(\underline{P}_{i t}^{g} v_{i t} \gamma_{i t}^{\text {lo }}+\bar{P}_{i t}^{g} v_{i t} \gamma_{i t}^{\text {up }}+\sum_{o \in \mathcal{O}_{i}} \bar{P}_{\text {oit }}^{g} \beta_{\text {oit }}^{\text {up }}\right) \\
& +\sum_{j \in \mathcal{J}} \sum_{t \in \mathcal{T}}\left(\underline{P}_{j t}^{d} \rho_{j t}^{\text {lo }}+\bar{P}_{j t}^{d} \rho_{j t}^{\text {up }}+\sum_{b \in \mathcal{B}_{j}} \bar{P}_{b j t}^{d} \mu_{b j t}^{\text {up }}\right) \\
& +\sum_{i \in \mathcal{I}}\left[P_{i 0}^{g} \xi_{i 1}+R_{i}^{\mathrm{up}} V_{i 0} \xi_{i 1}+R_{i}^{\mathrm{su}}\left(v_{i 1} \xi_{i 1}-V_{i 0} \xi_{i 1}\right)\right. \\
& \left.+\bar{P}_{i 1}^{g}\left(\xi_{i 1}-v_{i 1} \xi_{i 1}\right)\right] \\
& +\sum_{i \in \mathcal{I}} \sum_{t=2}^{n_{T}}\left[R_{i}^{\mathrm{up}} v_{i t-1} \xi_{i t}+R_{i}^{\mathrm{su}}\left(v_{i t} \xi_{i t}-v_{i t-1} \xi_{i t}\right)\right. \\
& \left.+\bar{P}_{i t}^{g}\left(\xi_{i t}-v_{i t} \xi_{i t}\right)\right] \\
& +\sum_{i \in \mathcal{I}}\left[P_{i 0}^{g} \sigma_{i 1}-R_{i}^{\mathrm{dn}} v_{i 1} \sigma_{i 1}-R_{i}^{\mathrm{sd}}\left(V_{i 0} \sigma_{i 1}-v_{i 1} \sigma_{i 1}\right)\right. \\
& \left.-\bar{P}_{i 1}^{g}\left(1-V_{i 0}\right) \sigma_{i 1}\right] \\
& -\sum_{i \in \mathcal{I}} \sum_{t=2}^{n_{T}}\left[R_{i}^{\mathrm{dn}} v_{i t} \sigma_{i t}+R_{i}^{\mathrm{sd}}\left(v_{i t-1} \sigma_{i t}-v_{i t} \sigma_{i t}\right)\right. \\
& \left.+\bar{P}_{i t}^{g}\left(\sigma_{i t}-v_{i t-1} \sigma_{i t}\right)\right] \\
& +\sum_{i \in \mathcal{I}} \sum_{t=1}^{n_{T}-1}\left[R_{i}^{\mathrm{sd}}\left(v_{i t} \epsilon_{i t}-v_{i t+1} \epsilon_{i t}\right)+\bar{P}_{i t}^{g} v_{i t+1} \epsilon_{i t}\right] .
\end{aligned}
$$


Expression (30) is nonlinear due to the presence of products of binary variables $v_{i t}$ and continuous Lagrange multipliers or dual variables associated with the lower-level problem. Based on [29], such nonlinearities can be equivalently recast as linear expressions. Thus, the nonlinear strong duality equality (30) is replaced by

$$
\begin{aligned}
& \sum_{j \in \mathcal{J}} \sum_{b \in \mathcal{B}_{j}} \sum_{t \in \mathcal{T}} C_{b j t}^{d} p_{b j t}^{d}-\sum_{i \in \mathcal{I}} \sum_{o \in \mathcal{O}_{i}} \sum_{t \in \mathcal{T}} C_{o i t}^{g} p_{o i t}^{g} \\
& =\sum_{t \in \mathcal{T}} \sum_{l \in \mathcal{L}_{t}^{+}} \bar{P}_{l}^{f} \phi_{l t}^{\mathrm{up}}-\sum_{t \in \mathcal{T}} \sum_{l \in \mathcal{L}_{t}^{-}} \bar{P}_{l}^{f} \phi_{l t}^{\mathrm{lo}} \\
& +\sum_{i \in \mathcal{I}} \sum_{t \in \mathcal{T}}\left(\underline{P}_{i t}^{g} a_{i t}+\bar{P}_{i t}^{g} b_{i t}+\sum_{o \in \mathcal{O}_{i}} \bar{P}_{\text {oit }}^{g} \beta_{\text {oit }}^{\text {up }}\right) \\
& +\sum_{j \in \mathcal{J}} \sum_{t \in \mathcal{T}}\left(\underline{P}_{j t}^{d} \rho_{j t}^{\mathrm{lo}}+\bar{P}_{j t}^{d} \rho_{j t}^{\text {up }}+\sum_{b \in \mathcal{B}_{j}} \bar{P}_{b j t}^{d} \mu_{b j t}^{\text {up }}\right) \\
& +\sum_{i \in \mathcal{I}}\left[P_{i 0}^{g} \xi_{i 1}+R_{i}^{\mathrm{up}} V_{i 0} \xi_{i 1}+R_{i}^{\mathrm{su}}\left(c_{i 1}-V_{i 0} \xi_{i 1}\right)\right. \\
& \left.+\bar{P}_{i 1}^{g}\left(\xi_{i 1}-c_{i 1}\right)\right] \\
& +\sum_{i \in \mathcal{I}} \sum_{t=2}^{n_{T}}\left[R_{i}^{\mathrm{up}} d_{i t}+R_{i}^{\mathrm{su}}\left(c_{i t}-d_{i t}\right)+\bar{P}_{i t}^{g}\left(\xi_{i t}-c_{i t}\right)\right] \\
& +\sum_{i \in \mathcal{I}}\left[P_{i 0}^{g} \sigma_{i 1}-R_{i}^{\mathrm{dn}} m_{i 1}-R_{i}^{\mathrm{sd}}\left(V_{i 0} \sigma_{i 1}-m_{i 1}\right)\right. \\
& \left.-\bar{P}_{i 1}^{g}\left(1-V_{i 0}\right) \sigma_{i 1}\right] \\
& -\sum_{i \in \mathcal{I}} \sum_{t=2}^{n_{T}}\left[R_{i}^{\mathrm{dn}} m_{i t}+R_{i}^{\mathrm{sd}}\left(r_{i t}-m_{i t}\right)+\bar{P}_{i t}^{g}\left(\sigma_{i t}-r_{i t}\right)\right] \\
& +\sum_{i \in \mathcal{I}} \sum_{t=1}^{n_{T}-1}\left[R_{i}^{\mathrm{sd}}\left(e_{i t}-k_{i t}\right)+\bar{P}_{i t}^{g} k_{i t}\right] \\
& \underline{\gamma}_{i t}^{\text {lo }} v_{i t} \leq a_{i t} \leq 0 ; \quad \forall i \in \mathcal{I}, \forall t \in \mathcal{T} \\
& \underline{\gamma}_{i t}^{\mathrm{lo}}\left(1-v_{i t}\right) \leq \gamma_{i t}^{\mathrm{lo}}-a_{i t} \leq 0 ; \quad \forall i \in \mathcal{I}, \forall t \in \mathcal{T} \\
& 0 \leq b_{i t} \leq \bar{\gamma}_{i t}^{\text {up }} v_{i t} ; \quad \forall i \in \mathcal{I}, \forall t \in \mathcal{T} \\
& 0 \leq \gamma_{i t}^{\text {up }}-b_{i t} \leq \bar{\gamma}_{i t}^{\text {up }}\left(1-v_{i t}\right) ; \quad \forall i \in \mathcal{I}, \forall t \in \mathcal{T} \\
& 0 \leq c_{i t} \leq \bar{\xi}_{i t} v_{i t} ; \quad \forall i \in \mathcal{I}, \forall t \in \mathcal{T} \\
& 0 \leq \xi_{i t}-c_{i t} \leq \bar{\xi}_{i t}\left(1-v_{i t}\right) ; \quad \forall i \in \mathcal{I}, \forall t \in \mathcal{T} \\
& 0 \leq d_{i t} \leq \bar{\xi}_{i t} v_{i t-1} ; \quad \forall i \in \mathcal{I}, \forall t=2 \ldots n_{T} \\
& 0 \leq \xi_{i t}-d_{i t} \leq \bar{\xi}_{i t}\left(1-v_{i t-1}\right) ; \quad \forall i \in \mathcal{I}, \forall t=2 \ldots n_{T} \\
& 0 \leq e_{i t} \leq \bar{\epsilon}_{i t} v_{i t} ; \quad \forall i \in \mathcal{I} \text {, } \\
& \forall t=1 \ldots n_{T}-1 \\
& 0 \leq \epsilon_{i t}-e_{i t} \leq \bar{\epsilon}_{i t}\left(1-v_{i t}\right) ; \quad \forall i \in \mathcal{I}, \\
& \forall t=1 \ldots n_{T}-1 \\
& 0 \leq k_{i t} \leq \bar{\epsilon}_{i t} v_{i t+1} ; \quad \forall i \in \mathcal{I}, \\
& \forall t=1 \ldots n_{T}-1 \\
& 0 \leq \epsilon_{i t}-k_{i t} \leq \bar{\epsilon}_{i t}\left(1-v_{i t+1}\right) ; \quad \forall i \in \mathcal{I}, \\
& \forall t=1 \ldots n_{T}-1 \\
& \underline{\sigma}_{i t} v_{i t} \leq m_{i t} \leq 0 ; \quad \forall i \in \mathcal{I}, \forall t \in \mathcal{T} \\
& \underline{\sigma}_{i t}\left(1-v_{i t}\right) \leq \sigma_{i t}-m_{i t} \leq 0 ; \quad \forall i \in \mathcal{I}, \forall t \in \mathcal{T}
\end{aligned}
$$

$$
\begin{array}{ll}
\underline{\sigma}_{i t} v_{i t-1} \leq r_{i t} \leq 0 ; & \forall i \in \mathcal{I}, \forall t=2 \ldots n_{T} \\
\underline{\sigma}_{i t}\left(1-v_{i t-1}\right) \leq \sigma_{i t}-r_{i t} \leq 0 ; & \forall i \in \mathcal{I}, \forall t=2 \ldots n_{T}
\end{array}
$$

where (31) is the linearized strong duality equality, whereas (32)-(33), (34)-(35), (36)-(37), (38)-(39), (40)-(41), (42)-(43), (44)-(45), and (46)-(47) correspond to the linearization of $v_{i t} \gamma_{i t}^{\mathrm{lo}}, v_{i t} \gamma_{i t}^{\mathrm{up}}, v_{i t} \xi_{i t}, v_{i t-1} \xi_{i t}, v_{i t} \epsilon_{i t}, v_{i t+1} \epsilon_{i t}, v_{i t} \sigma_{i t}$, and $v_{i t-1} \sigma_{i t}$, respectively.

Therefore, the mixed-integer linear equivalent for the lowerlevel problem includes (6)-(29) and (31)-(47).

\section{B. Linear Equivalent for the Energy Payment}

As above mentioned, and as a major distinctive modeling aspect with respect to previous works disregarding demand responsiveness [8], [15], the total payment for energy in (1) comprises bilinear terms involving the products of LMPs and consumption levels, which are both continuous decision variables. Here, we propose a novel and exact linearization of such bilinear terms based on duality theory of linear programming and KKT optimality conditions. From both modeling and computational perspectives, the proposed linearization is characterized by two relevant salient features over other methodologies addressing bilinear terms such as the binary expansion approach [42] and Schur's decomposition [43]: 1) rather than approximations, the resulting linear expressions are equivalent to the original bilinear products and 2) no additional binary variables are required.

The proposed transformation uses equalities (2) and (13), lower-level dual feasibility constraints (21) and (22), as well as the following complementary slackness conditions, which are associated with lower-level constraints (10) and (14):

$$
\begin{array}{rlrl}
\rho_{j t}^{\mathrm{lo}}\left(p_{j t}^{d}-\underline{P}_{j t}^{d}\right) & =0 ; & & \forall j \in \mathcal{J}, \forall t \in \mathcal{T} \\
\rho_{j t}^{\mathrm{up}}\left(p_{j t}^{d}-\bar{P}_{j t}^{d}\right) & =0 ; & \forall j \in \mathcal{J}, \forall t \in \mathcal{T} \\
\mu_{b j t}^{\mathrm{lo}}\left(p_{b j t}^{d}-0\right) & =0 ; & \forall j \in \mathcal{J}, \forall b \in \mathcal{B}_{j}, \forall t \in \mathcal{T} \\
\mu_{b j t}^{\mathrm{up}}\left(p_{b j t}^{d}-\bar{P}_{b j t}^{d}\right)=0 ; & \forall j \in \mathcal{J}, \forall b \in \mathcal{B}_{j}, \forall t \in \mathcal{T}
\end{array}
$$

where (48) and (49) are related to (10) while (50) and (51) correspond to (14).

From (48)-(51), the following equalities can be obtained:

$$
\begin{aligned}
\sum_{j \in \mathcal{J}} \sum_{t \in \mathcal{T}} \rho_{j t}^{\mathrm{lo}} p_{j t}^{d} & =\sum_{j \in \mathcal{J}} \sum_{t \in \mathcal{T}} \underline{P}_{j t}^{d} \rho_{j t}^{\mathrm{lo}} \\
\sum_{j \in \mathcal{J}} \sum_{t \in \mathcal{T}} \rho_{j t}^{\text {up }} p_{j t}^{d} & =\sum_{j \in \mathcal{J}} \sum_{t \in \mathcal{T}} \bar{P}_{j t}^{d} \rho_{j t}^{\text {up }} \\
\sum_{j \in \mathcal{J}} \sum_{b \in \mathcal{B}_{j}} \sum_{t \in \mathcal{T}} \mu_{b j t}^{\mathrm{lo}} p_{b j t}^{d} & =0 \\
\sum_{j \in \mathcal{J}} \sum_{b \in \mathcal{B}_{j}} \sum_{t \in \mathcal{T}} \mu_{b j t}^{\mathrm{up}} p_{b j t}^{d} & =\sum_{j \in \mathcal{J}} \sum_{b \in \mathcal{B}_{j}} \sum_{t \in \mathcal{T}} \bar{P}_{b j t}^{d} \mu_{b j t}^{\text {up }} .
\end{aligned}
$$

Using (2) and (21), the energy payment term in (1) can be recast as follows:

$$
\sum_{j \in \mathcal{J}} \sum_{t \in \mathcal{T}} \pi_{n(j) t} p_{j t}^{d}=\sum_{j \in \mathcal{J}} \sum_{t \in \mathcal{T}}\left(-\rho_{j t}^{\mathrm{lo}}-\rho_{j t}^{\mathrm{up}}-\delta_{j t}\right) p_{j t}^{d} .
$$


TABLE I

Dimensions of the ORIGinal BiLevel Model AND D-PM

\begin{tabular}{|c|c|c|c|}
\hline Model & $\#$ of constraints & $\begin{array}{c}\text { \# of binary } \\
\text { variables }\end{array}$ & $\begin{array}{l}\text { \# of real } \\
\text { variables }\end{array}$ \\
\hline $\begin{array}{l}\text { Original } \\
\text { (1)-(17) }\end{array}$ & $\begin{array}{c}n_{T}\left(n_{N}+1\right)+n_{I}+ \\
\sum_{t \in \mathcal{T}}\left(n_{L_{t}^{+}}+n_{L_{t}^{-}}\right)+ \\
n_{T}\left(9 n_{I}+3 n_{J}\right)+ \\
2 n_{T} \sum_{i \in \mathcal{I}} n_{O_{i}}+ \\
2 n_{T} \sum_{j \in \mathcal{J}} n_{B_{j}}\end{array}$ & $n_{I} n_{T}$ & $\begin{array}{c}n_{T} n_{N}+ \\
n_{T}\left(2 n_{I}+n_{J}\right)+ \\
n_{T} \sum_{i \in \mathcal{I}} n_{O_{i}}+ \\
n_{T} \sum_{j \in \mathcal{J}} n_{B_{j}}\end{array}$ \\
\hline $\begin{array}{c}\text { D-PM } \\
(59)-(60)\end{array}$ & $\begin{array}{c}n_{T}\left(n_{N}+1\right)-11 n_{I}+ \\
\sum_{t \in \mathcal{T}}\left(n_{L_{t}^{+}}+n_{L_{t}^{-}}\right)+ \\
n_{T}\left(34 n_{I}+4 n_{J}\right)+ \\
3 n_{T} \sum_{i \in \mathcal{I}} n_{O_{i}}+ \\
3 n_{T} \sum_{j \in \mathcal{J}} n_{B_{j}}+1\end{array}$ & $n_{I} n_{T}$ & $\begin{array}{c}n_{T}\left(n_{N}+1\right)-5 n_{I}+ \\
\sum_{t \in \mathcal{T}}\left(n_{L_{t}^{+}}+n_{L_{t}^{-}}\right)+ \\
n_{T}\left(16 n_{I}+4 n_{J}\right)+ \\
3 n_{T} \sum_{i \in \mathcal{I}} n_{O_{i}}+ \\
3 n_{T} \sum_{j \in \mathcal{J}} n_{B_{j}}\end{array}$ \\
\hline
\end{tabular}

In the same vein, using (13) and (22) in the last term of (56) yields

$$
\begin{aligned}
\sum_{j \in \mathcal{J}} \sum_{t \in \mathcal{T}} \pi_{n(j) t} p_{j t}^{d} & =\sum_{j \in \mathcal{J}} \sum_{t \in \mathcal{T}}\left(-\rho_{j t}^{\mathrm{lo}}-\rho_{j t}^{\mathrm{up}}\right) p_{j t}^{d} \\
& -\sum_{j \in \mathcal{J}} \sum_{b \in \mathcal{B}_{j}} \sum_{t \in \mathcal{T}}\left(\mu_{b j t}^{\mathrm{lo}}+\mu_{b j t}^{\mathrm{up}}-C_{b j t}^{d}\right) p_{b j t}^{d} .
\end{aligned}
$$

Finally, substituting (52)-(55) in (57), the total energy payment is cast as a linear equivalent as follows:

$$
\begin{aligned}
\sum_{j \in \mathcal{J}} \sum_{t \in \mathcal{T}} \pi_{n(j) t} p_{j t}^{d}= & \sum_{j \in \mathcal{J}} \sum_{t \in \mathcal{T}}\left(-\underline{P}_{j t}^{d} \rho_{j t}^{\mathrm{lo}}-\bar{P}_{j t}^{d} \rho_{j t}^{\text {up }}\right) \\
& -\sum_{j \in \mathcal{J}} \sum_{b \in \mathcal{B}_{j}} \sum_{t \in \mathcal{T}}\left(\bar{P}_{b j t}^{d} \mu_{b j t}^{\text {up }}-C_{b j t}^{d} p_{b j t}^{d}\right) .
\end{aligned}
$$

This linearization overcomes the difficulties of available approaches handling bilinear terms [42], [43] and allows the application of mixed-integer linear programming to the energy scheduling problem based on consumer payment minimization with price-responsive demand under marginal pricing. Therefore, this step constitutes the main methodological contribution of this paper.

\section{Single-Level Mixed-Integer Linear Equivalent}

The single-level equivalent of problem (1)-(17) is formulated as the following mixed-integer linear program, which is hereinafter referred to as D-PM:

$$
\begin{aligned}
& \underset{\substack{a_{i t}, b_{i t}, c_{i t}, d_{i t}, e_{i t}, k_{i t}, m_{i t}, p_{b j t}, p_{i t}^{d}, p_{i t}^{g},}}{\operatorname{sinimize}} \quad \sum_{j \in \mathcal{J}} \sum_{t \in \mathcal{T}}\left(-\underline{P}_{j t}^{d} \rho_{j t}^{\text {lo }}-\bar{P}_{j t}^{d} \rho_{j t}^{\text {up }}\right) \\
& p_{o i t}^{g}, r_{i t}, s_{i t}, v_{i t}, \alpha_{i t}, \\
& \beta_{\text {oit }}^{\text {lo }}, \beta_{o i t}^{\text {up }}, \gamma_{i t}^{\text {lo }}, \gamma_{i t}^{\text {up }}, \delta_{j t} \text {, } \\
& \epsilon_{i t}, \lambda_{t}, \mu_{b j t}^{\text {lo }}, \mu_{b j t}^{\text {up }}, \xi_{i t} \text {, } \\
& \pi_{n t}, \rho_{j t}^{\mathrm{lo}}, \rho_{j t}^{\text {up }}, \sigma_{i t}, \phi_{l t}^{\mathrm{lo},}, \phi_{l t}^{\text {up }} \quad+\sum_{i \in \mathcal{I}} \sum_{t \in \mathcal{T}} s_{i t} \\
& \begin{array}{l}
-\sum_{j \in \mathcal{J}} \sum_{b \in \mathcal{B}_{j}} \sum_{t \in \mathcal{T}}\left(\bar{P}_{b j t}^{d} \mu_{b j t}^{\text {up }}-C_{b j t}^{d} p_{b j t}^{d}\right) \\
+\sum_{i \in \mathcal{I}} \sum_{t \in \mathcal{T}} s_{i t}
\end{array}
\end{aligned}
$$

TABLE II

PRICES OF DEMAND BID BLOCKS (\$/MWh)

\begin{tabular}{crrr} 
& \multicolumn{3}{c}{ Block } \\
\cline { 2 - 4 } Consumer & 1 & \multicolumn{1}{c}{2} & 3 \\
\hline 1 & 50 & 20 & 18 \\
2 & 50 & 16 & 14 \\
3 & 50 & 12 & 10 \\
4 & 50 & 8 & 6 \\
5 & 50 & 7 & 5 \\
6 & 50 & 28 & 26 \\
7 & 50 & 25 & 20 \\
8 & 50 & 23 & 13 \\
9 & 50 & 22 & 15 \\
10 & 50 & 13 & 8 \\
$11-20$ & 50 & 20 & 18 \\
$21-30$ & 50 & 16 & 14 \\
$31-40$ & 50 & 12 & 10 \\
$41-50$ & 50 & 8 & 6 \\
$51-60$ & 50 & 7 & 5 \\
$61-70$ & 50 & 28 & 26 \\
$71-80$ & 50 & 25 & 20 \\
$81-86$ & 50 & 23 & 13 \\
$87-91$ & 50 & 22 & 15 \\
\hline
\end{tabular}

subject to

constraints (2)-(4), (6)-(29), and (31)-(47).

Table I provides the size of both the original bilevel problem (1)-(17) and the single-level mixed-integer linear equivalent D-PM (59), (60) in terms of the numbers of constraints, binary variables, and real variables. It is worth emphasizing that the number of minimum up and down time constraints depends on the initial statuses of generators. Therefore, the number of constraints listed in Table I for each model is an upper bound on the corresponding actual number of constraints. Note also that although the same number of binary variables is required by both models, the transformation process described in Sections III-A and III-B yields a larger number of constraints and real variables for D-PM.

\section{Numerical Results}

This section presents and discusses results from a case study based on the IEEE 118-bus system [44], [45]. This case study comprises 186 transmission lines, 54 generating units, and 91 consumers over a 24-h time span. The application of the findings reported in [36] to this system leads to the consideration of 412 line capacity constraints, i.e., only $4.6 \%$ of the whole set of line capacity constraints is required to equivalently model the effect of the transmission network. Generation data can be found in [45]. Three-block generation energy offers are obtained from the linearization of the quadratic production costs. Start-up offer prices are set equal to the start-up cost coefficients. It should be noted that generation data and offers remain unchanged over the time span. For illustration purposes, maximum demand bids are based on: 1) the nodal peak demands available in [44], which have been increased by $60 \%$; and 2) the hourly scaling factors reported in [46] for Wednesday of week 35. Moreover, it is assumed that consumers submit three-block demand bids priced as listed in Table II, with $\bar{P}_{1 j t}^{d}, \bar{P}_{2 j t}^{d}$, and $\bar{P}_{3 j t}^{d}$ equal to $90 \%, 5 \%$, and 5\% of the corresponding maximum demand bid level, respectively, 
TABLE III

PROBLEM Dimensions FOR THE 118-Bus SySTEM

\begin{tabular}{cccc}
\hline Model & \# of constraints & $\begin{array}{c}\text { \# of binary } \\
\text { variables }\end{array}$ & $\begin{array}{c}\text { \# of real } \\
\text { variables }\end{array}$ \\
\hline $\begin{array}{c}\text { Original } \\
(1)-(17)\end{array}$ & 42272 & 1296 & 18048 \\
\hline $\begin{array}{c}\text { D-PM } \\
(59)-(60)\end{array}$ & 86649 & 1296 & 63790 \\
\hline
\end{tabular}

TABLE IV

Payment Versus Declared Social Welfare (\$)

\begin{tabular}{ccc}
\cline { 2 - 3 } & D-PM & SWM \\
\hline Payment & 1903442.2 & 1985996.6 \\
Declared social welfare & 3736788.0 & 3786008.5 \\
\hline
\end{tabular}

and with $\underline{P}_{j t}^{d}=\bar{P}_{1 j t}^{d}, \forall j \in \mathcal{J}, \forall t \in \mathcal{T}$. Similar to producers, consumers do not modify the prices of their respective demand bid blocks over the time span. Table III presents the dimensions of both the original bilevel problem (1)-(17) and D-PM (59), (60) for this case study.

The results of D-PM are compared with those achieved by a conventional scheduling procedure based on the maximization of the declared social welfare [40]. This latter mechanism is hereinafter referred to as SWM. Both D-PM and SWM have been implemented on a Dell PowerEdge R910X64 with four Intel Xeon E7520 processors at $1.866 \mathrm{GHz}$ and $32 \mathrm{~GB}$ of RAM using CPLEX 12.1 [31] under GAMS 23.3 [47].

Using a stopping criterion for D-PM based on a $1 \%$ optimality gap, the proposed approach required $1231.3 \mathrm{~s}$ to attain the solution summarized in Table IV. According to current industry practice [41], [48], achieving a solution within a $1 \%$ optimality gap in around 20 min reveals an acceptable computational performance since the proposed algorithm is suitable to provide a solution that is very close to the optimum within a practical amount of time for a reasonably-sized problem. Moreover, the computational gain associated with the use of the network model described in [36] is also evidenced since CPLEX was unable to find a solution within a $1 \%$ optimality gap in less than $50000 \mathrm{~s}$ when the complete set of transmission capacity constraints was considered in the formulation of D-PM. Table IV also lists the results associated with the optimal solution to SWM, which was achieved in $4 \mathrm{~s}$. Note that the total payment by consumers drops from \$1985996.6 when a conventional market-clearing procedure is used down to $\$ 1903442.2$ when the alternative auction design is implemented. It is worth emphasizing that such substantial $4.2 \%$ reduction in the consumer payment is attained by moderately decreasing the declared social welfare by $1.3 \%$.

As shown in Fig. 1, D-PM and SWM yield almost identical consumption profiles, with slightly larger hourly awarded consumption levels under D-PM in most periods. This result reveals that, for this case study, a considerable consumer payment reduction can be achieved without significantly altering the consumption pattern. In addition, Fig. 1 depicts the hourly load-weighted average LMPs for D-PM and SWM. The load-weighted average LMP for time period $t$, denoted

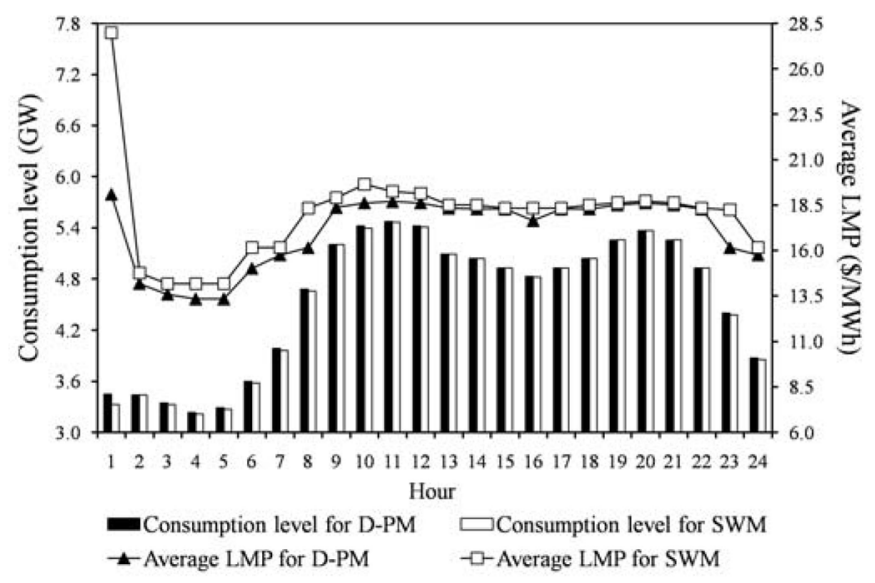

Fig. 1. Awarded consumption levels and load-weighted average LMPs.

TABLE V

IMPACT OF THE LEVEL OF DEMAND RESPONSIVENESS ON CONSUMER PAYMENT

\begin{tabular}{rc}
\hline $\bar{P}_{1 j t}^{d}(\%)$ & Payment (\$) \\
\hline $\bar{P}_{j t}^{d}$ & \\
\hline 100 & Infeasible \\
95 & 2019471.6 \\
90 & 1903442.2 \\
85 & 1808506.7 \\
80 & 1694624.1 \\
75 & 1601892.7 \\
\hline
\end{tabular}

as $\pi_{t}^{\text {av }}$, is computed as the sum over all buses of the products of the corresponding LMP and nodal consumption level divided by the total awarded consumption level in that period, i.e., $\pi_{t}^{\mathrm{av}}=\left(\sum_{n \in \mathcal{N}} \pi_{n t}\left(\sum_{j \in \mathcal{J}_{n}} p_{j t}^{d}\right) / \sum_{j \in \mathcal{J}} p_{j t}^{d}\right)$. It is worth noting that both curves follow the shape of the corresponding consumption profile, as expected under a marginal pricing scheme. Furthermore, it can be observed that loadweighted average LMPs yielded by D-PM are less than those resulting from SWM for all periods.

Finally, in order to assess the effect of the level of demandside participation, we have run D-PM for different values of $\bar{P}_{b j t}^{d}$, expressed as percentages of the corresponding maximum demand bid level, also with $\underline{P}_{j t}^{d}=\bar{P}_{1 j t}^{d}, \forall j \in \mathcal{J}, \forall t \in \mathcal{T}$. For the sake of simplicity, the percent values for $\bar{P}_{2 j t}^{d}$ and $\bar{P}_{3 j t}^{d}$ are identical. Thus, lower percent values for $\bar{P}_{1 j t}^{d}$ indicate larger levels of demand responsiveness. As illustrated in Table V, demandside participation is beneficial for the purposes of consumer payment minimization. For this particular case study, the case with inelastic demand, i.e., with a percent value for $\bar{P}_{1 j t}^{d}$ equal to $100 \%$, is infeasible due to network limitations.

\section{CONClusion}

Within the context of pool-based electricity markets, this paper has presented a novel and computationally efficient approach to incorporate price-responsive demand in multiperiod energy scheduling based on consumer payment minimization under marginal pricing. The salient feature of the proposed method lies in converting the resulting mixed-integer 
nonlinear bilevel program with bilinear terms into an equivalent mixed-integer linear programming problem.

The proposed approach has been successfully applied to a case study based on the IEEE 118-bus system with commercially available software. The major findings revealed by the numerical results are as follows.

1) High-quality solutions are attained in acceptable computing times.

2) The consumer payment is substantially reduced at the expense of moderately decreasing the declared social welfare.

3) The consumption pattern is not significantly altered with respect to that resulting from social welfare maximization.

4) Larger degrees of demand price responsiveness yield larger reductions in the consumer payment.

5) The use of the reduced and equivalent network model is beneficial for computational purposes.

Although a simple albeit practical characterization of demand-side participation has been presented in this paper, it should be noted that the main steps used in the proposed solution approach are readily applicable to incorporate other aspects of electricity consumption such as on/off consumption scheduling, limited energy consumption over the scheduling horizon, consumption ramping limits, load shifting, operational consumption cycles, and time-coupled price elasticity, among others. Such extension would require additional operational constraints as well as some extra notation to properly index variables and parameters. We recognize that the extended model needs further numerical studies.

Further research will be devoted to analyzing the effects of ac network constraints, additional demand-side operational constraints, and uncertainty sources such as wind power generation. Another interesting avenue of research is the analysis of tighter formulations and the application of decomposition techniques for the purposes of improved computational performance.

\section{REFERENCES}

[1] J. M. Jacobs, "Artificial power markets and unintended consequences," IEEE Trans. Power Syst., vol. 12, no. 2, pp. 968-972, May 1997.

[2] S. Hao, G. A. Angelidis, H. Singh, and A. D. Papalexopoulos, "Consumer payment minimization in power pool auctions," IEEE Trans. Power Syst., vol. 13, no. 3, pp. 986-991, Aug. 1998.

[3] J. Alonso, A. Trías, V. Gaitan, and J. J. Alba, "Thermal plant bids and market clearing in an electricity pool. Minimization of costs vs. minimization of consumer payments," IEEE Trans. Power Syst., vol. 14 no. 4, pp. 1327-1334, Nov. 1999.

[4] C. Vázquez, M. Rivier, and I. J. Pérez-Arriaga, "Production cost minimization versus consumer payment minimization in electricity pools," IEEE Trans. Power Syst., vol. 17, no. 1, pp. 119-127, Feb. 2002.

[5] S. Hao and F. Zhuang, "New models for integrated short-term forward electricity markets," IEEE Trans. Power Syst., vol. 18, no. 2, pp. 478-485, May 2003.

[6] Y. Chen et al., "Payment minimization auction with demand bids and partial compensation of startup costs for deregulated electricity markets," presented at the IEEE PES Gen. Meeting, San Francisco, CA, USA, Jun. 2005

[7] P. B. Luh et al., "Payment cost minimization auction for deregulated electricity markets using surrogate optimization," IEEE Trans. Power Syst., vol. 21, no. 2, pp. 568-578, May 2006.
[8] F. Zhao, P. B. Luh, J. H. Yan, G. A. Stern, and S.-C. Chang, "Payment cost minimization auction for deregulated electricity markets with transmission capacity constraints," IEEE Trans. Power Syst., vol. 23, no. 2, pp. 532-544, May 2008.

[9] F. Zhao, P. B. Luh, J. H. Yan, G. A. Stern, and S.-C. Chang, "Bid cost minimization versus payment cost minimization: A game theoretic study of electricity auctions," IEEE Trans. Power Syst., vol. 25, no. 1, pp. 181-194, Feb. 2010.

[10] P. B. Luh et al., "Payment cost minimization with demand bids and partial capacity cost compensations for day-ahead electricity auctions," in Economic Market Design and Planning for Electric Power Systems, J. Momoh and L. Mili, Eds. Hoboken, NJ, USA: Wiley, 2010, pp. 71-85.

[11] R. Fernández-Blanco, J. M. Arroyo, and N. Alguacil, "A unified bilevel programming framework for price-based market clearing under marginal pricing," IEEE Trans. Power Syst., vol. 27, no. 1, pp. 517-525, Feb. 2012

[12] Y. Xu, Q. Hu, and F. Li, "Probabilistic model of payment cost minimization considering wind power and its uncertainty," IEEE Trans. Sustain. Energy, vol. 4, no. 3, pp. 716-724, Jul. 2013.

[13] R. Jamalzadeh, M. M. Ardehali, S. H. Hosseinian, and M. Choopani, "Payment cost minimisation auction for deregulated electricity market using mixed-integer linear programming approach," IET Gener. Transmiss. Distrib., vol. 7, no. 8, pp. 907-918, Aug. 2013.

[14] R. Fernández-Blanco, J. M. Arroyo, and N. Alguacil, "Consumer payment minimization under uniform pricing: A mixed-integer linear programming approach," Appl. Energy, vol. 114, pp. 676-686, Feb. 2014.

[15] R. Fernández-Blanco, J. M. Arroyo, and N. Alguacil, "Networkconstrained day-ahead auction for consumer payment minimization," IEEE Trans. Power Syst., vol. 29, no. 2, pp. 526-536, Mar. 2014.

[16] L. Baringo and A. J. Conejo, "Transmission and wind power investment," IEEE Trans. Power Syst., vol. 27, no. 2, pp. 885-893, May 2012.

[17] E. Hirst, "Price-responsive retail demand: Key to competitive electricity markets," Pub. Util. Fortnightly, vol. 139, no. 5, pp. 34-41, Mar. 2001.

[18] D. S. Kirschen, "Demand-side view of electricity markets," IEEE Trans. Power Syst., vol. 18, no. 2, pp. 520-527, May 2003.

[19] C.-L. Su and D. Kirschen, "Quantifying the effect of demand response on electricity markets," IEEE Trans. Power Syst., vol. 24, no. 3, pp. 1199-1207, Aug. 2009.

[20] A. J. Conejo, J. M. Morales, and L. Baringo, "Real-time demand response model," IEEE Trans. Smart Grid, vol. 1, no. 3, pp. 236-242, Dec. 2010.

[21] M. Parvania, M. Fotuhi-Firuzabad, and M. Shahidehpour, "Optimal demand response aggregation in wholesale electricity markets," IEEE Trans. Smart Grid, vol. 4, no. 4, pp. 1957-1965, Dec. 2013.

[22] A. Safdarian, M. Fotuhi-Firuzabad, and M. Lehtonen, "Integration of price-based demand response in DisCos' short-term decision model," IEEE Trans. Smart Grid, vol. 5, no. 5, pp. 2235-2245, Sep. 2014.

[23] A. Safdarian, M. Fotuhi-Firuzabad, and M. Lehtonen, "A distributed algorithm for managing residential demand response in smart grids," IEEE Trans. Ind. Informat., vol. 10, no. 4, pp. 2385-2393, Nov. 2014.

[24] A. Safdarian, M. Fotuhi-Firuzabad, and M. Lehtonen, "A medium-term decision model for DisCos: Forward contracting and TOU pricing," IEEE Trans. Power Syst., vol. 30, no. 3, pp. 1143-1154, May 2015.

[25] F. C. Schweppe, M. C. Caramanis, R. D. Tabors, and R. E. Bohn, Spot Pricing of Electricity. Norwell, MA, USA: Kluwer, 1988.

[26] E. Litvinov, "Design and operation of the locational marginal pricesbased electricity markets," IET Gener. Transmiss. Distrib., vol. 4, no. 2 , pp. 315-323, Feb. 2010.

[27] J. F. Bard, Practical Bilevel Optimization. Algorithms and Applications. Norwell, MA, USA: Kluwer, 1998.

[28] J. M. Arroyo, "Bilevel programming applied to power system vulnerability analysis under multiple contingencies," IET Gener. Transmiss. Distrib., vol. 4, no. 2, pp. 178-190, Feb. 2010.

[29] C. A. Floudas, Nonlinear and Mixed-Integer Optimization: Fundamentals and Applications. New York, NY, USA: Oxford Univ. Press, 1995.

[30] G. L. Nemhauser and L. A. Wolsey, Integer and Combinatorial Optimization. Hoboken, NJ, USA: Wiley, 1999.

[31] (2015). The IBM ILOG CPLEX Website. [Online]. Available: http:// www-01.ibm.com/software/commerce/optimization/cplex-optimizer 
[32] Market Operator of the Iberian Electricity Market. (May 2014). Daily and Intraday Electricity Market Operating Rules. [Online]. Available: http://www.omel.es/files/20140509_reglas_v11_ingles.pdf

[33] California ISO. (Oct. 2014). Bid and Self-Schedule Submission for All CAISO Markets. [Online]. Available: http://www.caiso.com/Documents/ Section30_BidAndSelfScheduleSubmissionForAllCAISOMarkets_Oct1_ 2014.pdf

[34] ISO New England, Inc. (Dec. 2014). ISO New England Manual for Market Operations: Manual M-11. [Online]. Available: http://www.iso-ne.com/static-assets/documents/2014/12/m_11_market_ operations_revision_48_12_03_14.doc

[35] A. Gómez-Expósito, A. J. Conejo, and C. Cañizares, Electric Energy Systems: Analysis and Operation. Boca Raton, FL, USA: CRC Press, 2008.

[36] Q. Zhai, X. Guan, J. Cheng, and H. Wu, "Fast identification of inactive security constraints in SCUC problems," IEEE Trans. Power Syst., vol. 25, no. 4, pp. 1946-1954, Nov. 2010.

[37] M. Carrión and J. M. Arroyo, "A computationally efficient mixed-integer linear formulation for the thermal unit commitment problem," IEEE Trans. Power Syst., vol. 21, no. 3, pp. 1371-1378, Aug. 2006.

[38] Federal Energy Regulatory Commission. (Nov. 2011). Recent ISO Software Enhancements and Future Software and Modeling Plans. [Online]. Available: http://www.ferc.gov/ industries/electric/indus-act/rto/rto-iso-soft-2011.pdf

[39] M. Shahidehpour, H. Yamin, and Z. Li, Market Operations in Electric Power Systems: Forecasting, Scheduling, and Risk Management. Hoboken, NJ, USA: Wiley, 2002.

[40] A. L. Motto, F. D. Galiana, A. J. Conejo, and J. M. Arroyo, "Networkconstrained multiperiod auction for a pool-based electricity market," IEEE Trans. Power Syst., vol. 17, no. 3, pp. 646-653, Aug. 2002.

[41] R. Jiang, J. Wang, M. Zhang, and Y. Guan, "Two-stage minimax regret robust unit commitment," IEEE Trans. Power Syst., vol. 28, no. 3, pp. 2271-2282, Aug. 2013.

[42] M. V. Pereira, S. Granville, M. H. C. Fampa, R. Dix, and L. A. Barroso, "Strategic bidding under uncertainty: A binary expansion approach," IEEE Trans. Power Syst., vol. 20, no. 1, pp. 180-188, Feb. 2005.

[43] R. A. Horn and C. R. Johnson, Matrix Analysis, 2nd ed. New York, NY, USA: Cambridge Univ. Press, 2012.

[44] Power Systems Test Case Archive, Dept. Elect. Eng., Univ. Washington, Seattle, WA, USA, 2015. [Online]. Available: http://www.ee.washington.edu/research/pstca

[45] (2015). IEEE 118-Bus System. [Online]. Available: http://motor.ece.iit.edu/data/Data_118_Bus.pdf

[46] C. Grigg et al., "The IEEE Reliability Test System-1996. A report prepared by the Reliability Test System Task Force of the Application of Probability Methods Subcommittee," IEEE Trans. Power Syst., vol. 14, no. 3, pp. 1010-1020, Aug. 1999.

[47] (2015). The GAMS Development Corporation Website. [Online]. Available: http://www.gams.com

[48] R. Sioshansi, R. O’Neill, and S. S. Oren, "Economic consequences of alternative solution methods for centralized unit commitment in dayahead electricity markets," IEEE Trans. Power Syst., vol. 23, no. 2, pp. 344-352, May 2008.
Ricardo Fernández-Blanco (S'10-M'15) received the Ingeniero Industrial and Ph.D. degrees in electrical engineering from the Universidad de Castilla-La Mancha, Ciudad Real, Spain, in 2009 and 2014, respectively.

$\mathrm{He}$ is currently a Post-doctoral Researcher with the University of Washington, Seattle, WA, USA. His current research interests include energy and power systems, bilevel programming, and electricity markets.

José M. Arroyo (S'96-M'01-SM'06) received the Ingeniero Industrial degree from the Universidad de Málaga, Málaga, Spain, in 1995, and the Ph.D. degree in power systems operations planning from the Universidad de Castilla-La Mancha, Ciudad Real, Spain, in 2000.

$\mathrm{He}$ is currently a Full Professor of Electrical Engineering with the Universidad de Castilla-La Mancha. His current research interests include operations, planning, and economics of power systems, as well as optimization and parallel computation.

Natalia Alguacil (S'97-M'01-SM'07) received the Ingeniero en Informática degree from the Universidad de Málaga, Málaga, Spain, in 1995, and the Ph.D. degree in power systems operations and planning from the Universidad de Castilla-La Mancha, Ciudad Real, Spain, in 2001.

She is currently an Associate Professor of Electrical Engineering with the Universidad de Castilla-La Mancha. Her current research interests include operations, planning, and economics of power systems, as well as optimization.

Xiaohong Guan (M'93-SM'95-F'07) received the B.S. and M.S. degrees in automatic control from Tsinghua University, Beijing, China, in 1982 and 1985, respectively, and the Ph.D. degree in electrical engineering from the University of Connecticut, Storrs, CT, USA, in 1993.

$\mathrm{He}$ was a Senior Consulting Engineer with Pacific Gas and Electric Company, San Francisco, CA, USA, from 1993 to 1995. He visited the Division of Engineering and Applied Science, Harvard University, Cambridge, MA, USA, from 1999 to 2000. Since 1995, he has been with the Systems Engineering Institute, Xi'an Jiaotong University, Xi'an, China, and was appointed as the Cheung Kong Professor of Systems Engineering in 1999, and the Dean of the School of Electronic and Information Engineering in 2008. Since 2001, he has been the Director of the Center for Intelligent and Networked Systems, Tsinghua University, where he served as the Head of the Department of Automation from 2003 to 2008. His current research interests include scheduling of power and manufacturing systems, bidding strategies for deregulated electric power markets, and security of complex networked systems. 